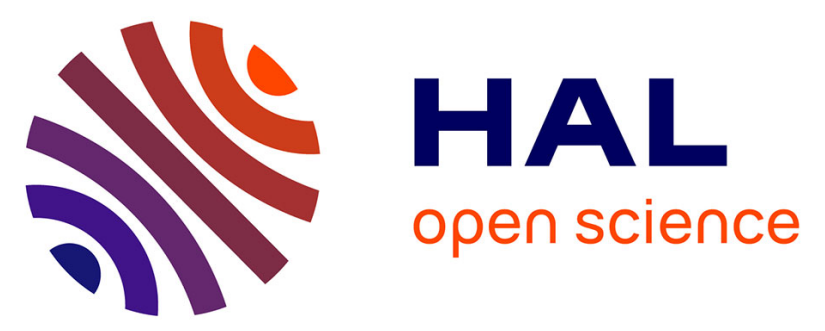

\title{
Computational insights about the dynamic behavior for the inclusion process of deprotonated and neutral aspirin in beta-cyclodextrin
}

Belgacem Bezzina, Rayenne Djémil, Djamel Eddine Khatmi, Stéphane Humbel, Yannick Carissan

\section{To cite this version:}

Belgacem Bezzina, Rayenne Djémil, Djamel Eddine Khatmi, Stéphane Humbel, Yannick Carissan. Computational insights about the dynamic behavior for the inclusion process of deprotonated and neutral aspirin in beta-cyclodextrin. Journal of Inclusion Phenomena and Macrocyclic Chemistry, 2018, 92 (1-2), pp.115-127. 10.1007/s10847-018-0822-0 . hal-02168191v2

\section{HAL Id: hal-02168191 \\ https://hal.science/hal-02168191v2}

Submitted on 12 Apr 2021

HAL is a multi-disciplinary open access archive for the deposit and dissemination of scientific research documents, whether they are published or not. The documents may come from teaching and research institutions in France or abroad, or from public or private research centers.
L'archive ouverte pluridisciplinaire HAL, est destinée au dépôt et à la diffusion de documents scientifiques de niveau recherche, publiés ou non, émanant des établissements d'enseignement et de recherche français ou étrangers, des laboratoires publics ou privés. 


\title{
Computational insights about the dynamic behavior for the inclusion process of deprotonated and neutral aspirin in $\beta$-cyclodextrin
}

\author{
Belgacem Bezzina, ${ }^{\mathrm{a}, \mathrm{b}}$ Rayenne Djémil, ${ }^{\mathrm{a}}$ Djamel eddine Khatmi, ${ }^{\mathrm{c}}$ Stéphane Humbel, ${ }^{\mathrm{c}}$ \\ Yannick Carissan, ${ }^{\mathrm{c}}$ \\ a - Laboratory of Computational Chemistry and Nanostructures, University of 08 May 45 \\ Guelma, Algeria \\ b- Research Center in Industrial Technologies (CRTI), P.O.BOX 64, Chéraga 16014, Algiers, \\ Algeria \\ c- Aix Marseille Univ, CNRS, Centrale Marseille, iSm2, Marseille, France \\ *corresponding author: khatmi.djameleddine@gmail.com
}

KEYWORDS: Aspirin. Cyclodextrin. Inclusion complexes. Molecular dynamic simulation. Umbrella sampling. Hydrogen bonding. 
ABSTRACT: Molecular dynamics was used to study the inclusion of neutral and deprotonated aspirin into the $\beta$-cyclodextrin $(\beta-\mathrm{CD})$ cavity. The molecular dynamic simulation allows following the time dependent behavior of the formation of the inclusion complex. For both complexes, we find a reasonable and a realistic pattern of the complexation. The calculations show a single pathway consisting of a no reversible binding process leading to the complexation of aspirin. Whereas for deprotonated aspirin it has been observed a reversible binding, in which one way leads to the binding form, and the reverse way to the unbinding form. Throughout the simulation, the penetration of aspirin (ASA) or deprotonated aspirin (ASA-) inside the cavity occurs only with a phenyl ring entering first through the wider or narrower rim. The determination of free energy using unbiased and biased simulations of the corresponding inclusion processes gives more favorable inclusion process of aspirin than deprotonated aspirin. The inclusion of the guest molecule is found deeply embedded within ASA: $\beta$-CD complex whereas it is partial in $\mathrm{ASA}^{-}: \beta-\mathrm{CD}$ complex. Also, the orientation A of both complexes is found more favorable of ca. $1.9 \mathrm{kcal} / \mathrm{mol}$, and of ca. $0.8 \mathrm{kcal} / \mathrm{mol}$, respectively for neutral and deprotonated complex. Aspirin molecule establish one H-bond between the hydrogen carboxylic atom and one oxygen atom of primary hydroxyl group of $\beta-\mathrm{CD}$; this $\mathrm{H}$-bond is detected during about $20 \%$ of the simulation period. In addition, we found that water molecules in the first solvation layer are implied with hydrogen carboxylic atom and the keto oxygen atoms within $\mathrm{H}$ bonds. While, water molecules of the second solvation layer is in interact with the $\mathrm{O} 1$ and $\mathrm{O} 2$ oxygen atoms of aspirin. Accordingly, based on the obtained results we can consider that the hydrophobic/hydrophilic interactions are the most important driving forces of the complexation assisted by stabilizing H-bonds. 


\section{1- INTRODUCTION}

An inclusion complex - a combination of two or more species - is based on a non-covalent interaction between the host and guest molecules and their stability depends on the size and complementary forms of host and guest molecules. Additionally, the external medium and environmental conditions can also play a role [1-4].

$\beta$-cyclodextrin $(\beta-C D)$ is a cyclic oligosaccharide derived by enzymatic hydrolysis of common starch. Due to its peculiar chemical structure, constituted of an external hydrophilic surface and a hydrophobic cavity, $\beta-\mathrm{CD}$ can form inclusion complexes with a variety of organic molecules, thereby improving some of their properties, such as solubility, stability and bio-availability [5-7]. The main forces involved in the complexation process are electrostatics, van der Waals, hydrophobic interactions, hydrogen bonding and charge transfer interactions [8-10]. The complexation process in solution comprises several steps, which we briefly summarize here: at first, as the guest molecule approaches the $\beta-\mathrm{CD}$ cavity, it is released from the hydration layer which surrounded it, and conjointly the water molecules which are inside the cavity are released outside. Then, the guest molecule penetrates inside the $\beta-\mathrm{CD}$ cavity giving a chemical complex stabilized only by non-covalent interactions.

If the guest molecule finds a large stability inside the cavity, it will remain there for a long time, and the process can be considered as a no reversible binding. Otherwise, without large stabilizing interactions with the host molecule inside the cavity, it will be released and water molecules would return in the cavity. However, the guest molecule can return inside the cavity so the process is considered as a reversible binding which can occur repeatedly [8].

Several factors may influence the inclusion complex formation, such as the type of CD, cavity size, $\mathrm{pH}$ and ionization state, temperature and method of preparation [11-12].

The molecular encapsulation process has constituted a major area of expertise and investigation within the framework of supramolecular chemistry. It became the subject of intense experimental and theoretical studies because the detailed knowledge of the dynamics of complexation constitutes the basis of its use as a drug carrier [13-14].

Acetylsalicylic acid (ASA), also known by the trade name aspirin, is one of the most known, used and popular drugs. It belongs to a group of medication called non-steroidal antiinflammatory drug (NSAIDs); it is often used to treat pain [15], fever [16], inflammation [17], to 
help prevent heart attacks [18], blood clot formation [19], and in a certain type of cancer, as colorectal or pancreatic cancer $[20,21]$.

The spontaneous hydrolysis of aspirin varies markedly with $\mathrm{pH}$. At $\mathrm{pH}$ below 2.5 aspirin is in its neutral form, whereas, at higher $\mathrm{pH}$ it becomes increasingly deprotonated. Its rate of absorption in the stomach, which has a $\mathrm{pH}$ of 1.5 to 3.5 , depends on its form. Thus, the majority of the biological activities, attributed to the neutral form are due to its lipophilic nature, which facilitates its absorption through the stomach wall, and its passing into the bloodstream [22, 23]. In many cases their use at higher doses can generate bad side effects, notably gastric irritation, gastrointestinal ulcers, hematemesis, tinnitus and stomach bleeding $[24,25]$. These inconveniences are due, in particular, to its low solubility in water estimated to $1 \mathrm{~g} / 300 \mathrm{ml}$ [26], caused by its rapid decomposition in two acids, salicylic and acetic [27].

Several methods are used to overcome these inconveniences such as its use as a pro drug or microencapsulation. The microencapsulation system, also named inclusion complex, is constituted of two molecules forming a single entity in which the molecule called "guest" is trapped in the cavity of another one, called "host"; and the system is stabilized only by noncovalent interactions $[28,29]$.

The application of the microencapsulation technique, allows improving the apparent solubility of poorly soluble molecules, protecting the fragile ones from unfavorable conditions and enhancing their therapeutic properties, such as its use as a drug delivery system (DDS) [30-34].

Many authors have studied the complexation process of aspirin with $\beta-C D$ using various techniques such as X-ray (RX) [35], spectroscopic methods [36, 37], ultrasonic relaxation [38], and isothermal calorimetric titration [23].

Computational chemistry methods can give appropriate answers because they have already had a great success in solving several problems related to the geometry, the physical chemistry, and the driving forces of the complexation [39-41].

The number of published papers in the past decade on this topic has widely increased, showing the evidence of a passion, attesting the mastery and the growing knowledge on the subject [4244]. Furthermore, while the $\mathrm{pH}$ is of primary importance for its biological effect, to the best of our knowledge, there are no reports in the literature on the evolution of the complexation of $\beta$ CD with ASA vs. ASA-. 
For this purpose, we describe in this article, a computational study on the complexation process between $\mathrm{ASA} / \mathrm{ASA}^{-}$and $\beta-\mathrm{CD}$ using molecular dynamics simulation. Then, we shall focus our attention on the dynamic of the formation of H-bonds during the simulation in order to giving a dynamic picture of the complexation process.

\section{2- METHODS}

The initial structure of $\beta-C D$ was built with the help of the graphical interface of ChemBioOffice (version 10, Cambridge software) [45], and the geometry of ASA was extracted from a PubChem Compound Database [46] (Figure 1). Thus, partial charges and the optimized geometries at B3LYP/6-31G(d) for guests and $\beta \mathrm{CD}$ molecules are given by the automated force field topology builder (ATB) [https://atb.uq.edu.au/.] [47].

However, the center of mass of aspirin and $\beta \mathrm{CD}$ were generated by Gromacs program and the radius of gyration is calculated using the following equation:

$$
\mathrm{R}_{\mathrm{g}}=\left(\frac{\sum\left|\mathrm{r}_{\mathrm{i}}\right|^{2} \mathrm{x}_{\mathrm{i}}}{\sum \mathrm{m}_{\mathrm{i}}}\right)^{2}
$$

Where $m_{i}$ is the mass of atom $i$ and $r_{i}$ the position of atom $i$ with of the respect to the center of mass of the molecule. The center of mass (COM) of the $\beta \mathrm{CD}$ was set as the origin of the reference system (0). Two possible orientations were considered. When phenyl group is located on the wider rim is called "orientation A" and when it is located on the narrower rim is called "orientation B". 
<smiles>CC(=O)Oc1ccccc1C(=O)O</smiles>
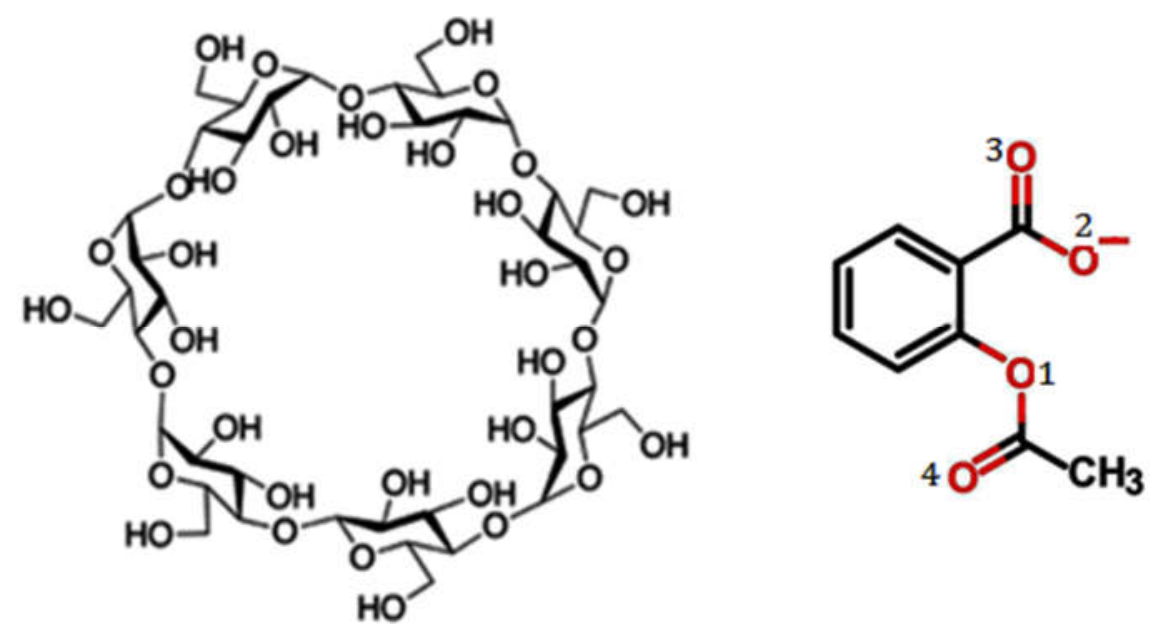

Figure 1. ASA, ASA- and $\beta-\mathrm{CD}$ molecular structures

\subsection{Molecular dynamics}

\subsubsection{MD simulations}

First, $\beta-C D$ is fixed in the center of XYZ coordinate system. Then, ASA/ASA ${ }^{-}$is placed on the $\mathrm{X}$-coordinate axe at a distance equal to $10 \AA$ from the center of $\beta-\mathrm{CD}$ (Figure 1,2).

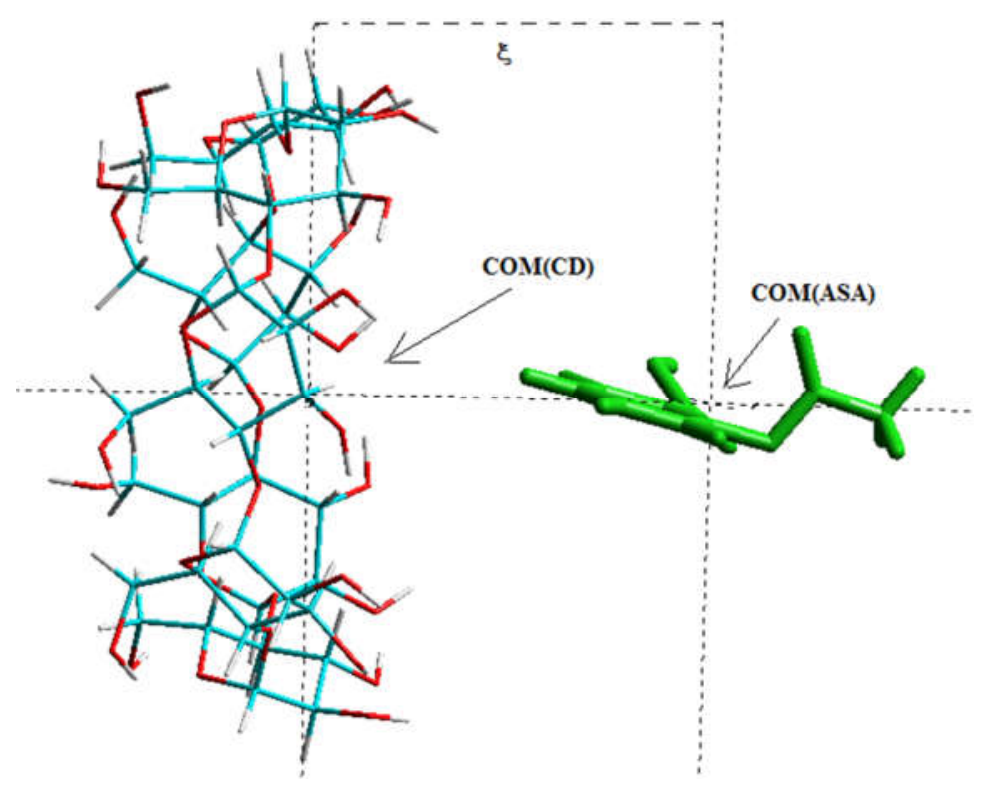

Figure 2. The initial geometry of ASA and $\beta-\mathrm{CD}$ molecules at the beginning of both simulations. 
The force field parameters for both molecules were generated using the automated topology builder (ATB) in the framework of GROMOS96 54A7 force field [48].

All simulations were carried out using the GROMACS package version 5.2.1 into an identical box containing 2638 water molecules represented by a simple point charge (SPC) model [49, 50]. The simulations were performed in the isobaric-isothermal ensemble (NPT) with a constant pressure of 1 bar and a temperature of $298 \mathrm{~K}$ controlled by the Berendsen thermostat. The electrostatic interactions were treated by the particle mesh Ewald (PME) method with a cutoff distance of $1.2 \mathrm{~nm}$; and the van der Waals interactions were modeled using a cutoff of $1.4 \mathrm{~nm}$. The whole system was relaxed using the steepest descent algorithm. The position of the guest molecule is obviously altered after the equilibration steps. The classical Newton's equations of motion were integrated using the Leap-Frog algorithm and the Berendsen weak-coupling method. All simulations were carried out for $1000 \mathrm{~ns}$, which is long enough for the system to reach equilibrium. The trajectory coordinates were recorded every 500 steps with a time step of 1 fs. The simulations were made using from 16 to 32 microprocessors and each one lasts 10 days at the most. The percent of time spend inside and outside the cavity is calculated by inspecting the distance between the two centroïds during the simulations. All values higher than $6.5 \AA$ are considered outside the cavity, whereas the values equal or less $6.5 \AA$ are considered inside the cavity. For each case we calculate the average of the three independent runs.

The kinetics of binding between a single molecule and its ligand is usually described by as a simple two-state model,

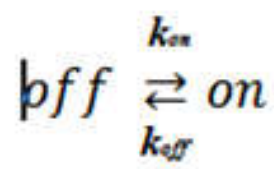

Where state "off" indicates that the molecule is free and state "on" indicates that the molecule is bound to a ligand, and the transition rate from "off" to "on" is proportional to the ligand concentration due to the law of mass action [51].

We can write the eq. 1 for ASA / ASA- reaction with $\beta-\mathrm{CD}$ as,

$$
A S A / A S A-+\beta-C D \underset{k_{\text {eff }}}{\stackrel{k_{e s}}{\rightleftarrows}} A S A: \beta-C D / A S A-: \beta-C D \cdots \cdots \cdots
$$


One can then obtain the apparent rate constants $k_{\text {on }}$ and $k_{\text {off }}$ from the residence time, on and off respectively as:

$$
k_{\text {on }}=\frac{1}{\text { average unbound time } X[\text { solute }]} \cdots \cdots \cdots
$$

And,

$$
k_{\text {off }}=\frac{1}{\text { average bound time }} \cdots \cdots \cdots
$$

The thermodynamics and kinetic are related by the equation:

$$
K=\frac{k_{o n}}{k_{o f f}} \cdots \cdots \cdots
$$

The binding free energy can be obtained from the constant $K$ that:

$$
\Delta G^{\circ}=-R T \ln K
$$

\subsubsection{Umbrella sampling}

The stability of the inclusion complexes can be assessed by computing the free energy change of the inclusion process using biased molecular dynamics of umbrella sampling (US) in addition to the weighted histogram analysis (WHAM) method implemented in the WHAM program [52]. In this technique, the potentials of mean force (PMF) were calculated as a function of the coordinate distance $(\xi)$ which is defined as the distance between the centroïds of mass of ASA/ $\mathrm{ASA}^{-}$and the point 0 , the centroïds of mass of $\beta$-CD. Each system is constrained to a narrow range of reaction coordinates using an external quadratic polarization force with a force constant of $\mathrm{k}=1000 \mathrm{kcal} \mathrm{mol}^{-1} \AA^{-2}$. The values of $\xi$ ranged from -10.0 to $10.0 \AA$ in $0.25 \AA$ intervals. Thus, 80 windows were used to cover this interval and then a simulation of $10 \mathrm{~ns}$ was performed for each window.

The distance data were collected every $1.0 \mathrm{ps}$, then the data from all the simulation windows were combined, and finally the weighted Histogram Analysis Method (WHAM) was used to analyze the results.

\section{3- Results and discussions}

\subsection{Molecular dynamics simulation}


Three independent molecular dynamics simulations of $1000 \mathrm{~ns}$ were performed to describe the inclusion process of $\mathrm{ASA} / \mathrm{ASA}^{-}$with $\beta-\mathrm{CD}$ at the molecular level. The simulations with ASA are reported in Figure 3, with the labels S1, S2, S3 and those of ASA ${ }^{-}$are in Figure 4 (S'1, S'2, and S'3). In this study, the complex is considered closely related if the distances between the two centroïds of mass of ASA/ASA ${ }^{-}$and $\beta-C D$ are less than $3 \AA$. Structures with distances between 3 and $6.5 \AA$ are considered as linked, however, ASA/ASA- is considered "unbound" if the distance becomes larger than $6.5 \AA$. Also, we chose to measure the distance between H5 and H20 atoms during the simulation in order to locate the conformations of A or B orientation and accordingly to determine the lifetime in each orientation.

\subsubsection{The kinetics of inclusion of $A S A$ with $\beta-C D$}

Throughout the simulation, the inclusion process is described through the analysis of the variation of the distances between the two centroïds of mass of ASA and $\beta-C D$ (Figure 3). In all three simulations it was observed that the penetrations and exits of ASA in and out of the cavity can occur. As it can be seen in Figure 3, three interesting facts are emerging from the three simulations: (i) a rapid penetration of ASA, which can last only a few ns, followed by a long stay inside the cavity; it remains there deeply embedded until the end of the simulation; except of the S2 simulation where around 400 ps ASA briefly leaves the cavity and returns rapidly inside. (ii) also, there are some regions where there are more fluctuations, indicating that ASA moves in these regions freely inside the cavity, and sometimes get out of the cavity (iii) changes of the ASA orientation inside the $\beta-\mathrm{CD}$ cavity occur during the simulations, from $\mathrm{A}$ to $\mathrm{B}$ or the reverse, these changes of orientation are made via a few unbound forms. 


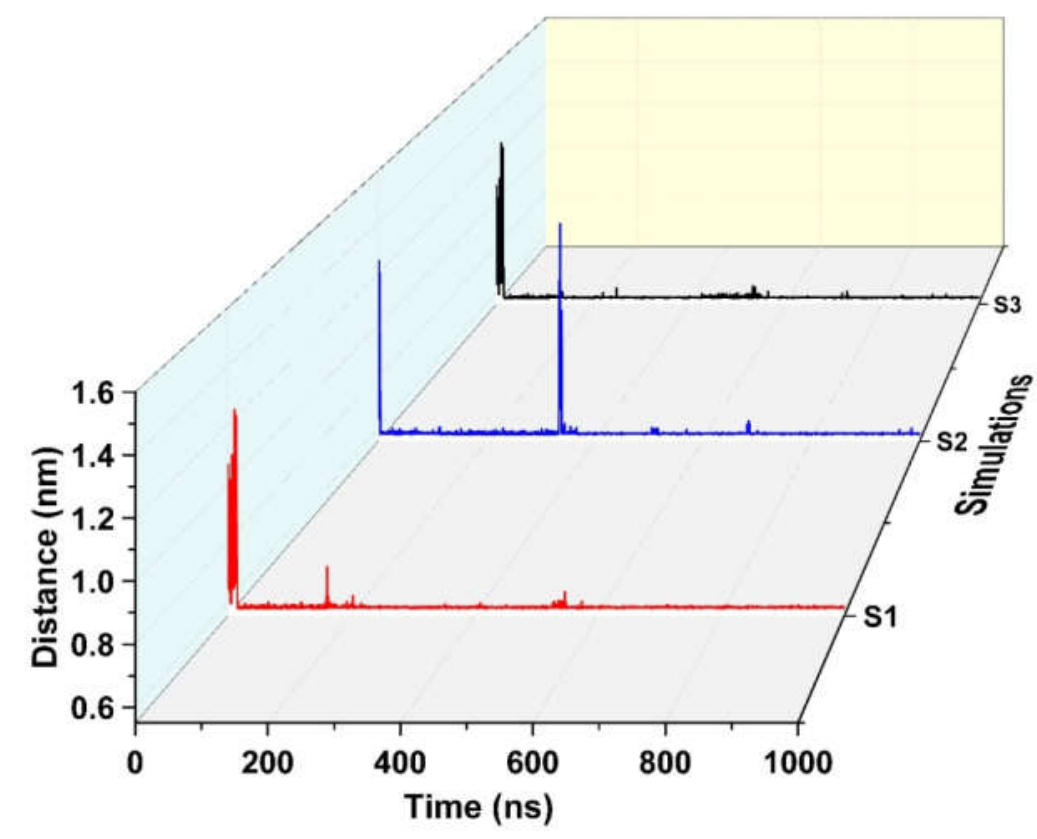

Figure 3. The plot of the variation of the distance between the two centroïds of mass of ASA and $\beta-\mathrm{CD}$ during the three simulations.

From these simulations, four key points are highlighted: (i) mostly the complexation process is a no reversible binding (i.e., once ASA penetrates into the cavity, it is no longer available in the medium); (ii) ASA can penetrated by the two entrances with the phenyl ring first ; (iii) the inclusion complex is favorable in aqueous medium because ASA spent much more time inside the cavity, 98\%(S1), 98\% (S2) and 99\% (S3) of the simulation period than outside; (iv) the orientation $\mathrm{A}$ is more favorable than the $\mathrm{B}$ one, because the lifetime of the orientation $\mathrm{A}$ is larger than that of orientation B: the orientation A lasts $81 \%(\mathrm{~S} 1), 70 \%(\mathrm{~S} 2)$ and $84 \%(\mathrm{~S} 3)$ of the simulation period while B lasts $17 \%(\mathrm{~S} 1), 28 \%(\mathrm{~S} 2)$ and $15 \%(\mathrm{~S} 3)$ of the simulation period. From a structural viewpoint, the conformational characteristic of the structures in orientation $\mathrm{A}$ ASA shows an inclusion deeply embedded inside the cavity, whereas in the orientation B only the phenyl ring is included and the functional groups stay in secondary rim establishing H-bonds with $\beta-\mathrm{CD}$. 


\subsubsection{The kinetics of inclusion of $A S A^{-}$with $\beta-C D$}

The simulations are labelled S'1, S'2, S'3, and they are shown on Figure 4. Throughout all three simulations it was observed, that the phenyl group penetrates first through the wider or narrower side. The penetration of $\mathrm{ASA}^{-}$is incomplete: only the phenyl group enters the $\beta-\mathrm{CD}$ cavity, whereas the acetyl and carboxylate groups remain outside.

As can be seen from Figure 4, the variation of the distance between the center of mass of ASA $^{-}$ and $\beta-C D$ is very similar from one simulation to another: $S^{\prime} 1$ resembles $S^{\prime} 2$ and $S^{\prime} 3$ is alike.

In each simulation, it was observed multiple entrances and exits of $\mathrm{ASA}^{-}$of $\beta-\mathrm{CD}$ cavity. At each time, the guest molecule remains only a small moment inside the cavity with the exception of the periods [300-450] for $S^{\prime} 1$, [200-310] for $S^{\prime} 2$ and [700-790] ns for $S^{\prime} 3$ where it passes more than 90 ns inside the cavity.

Overall, these three simulations are less in favor of an inclusion process because the guest molecule spent more time outside $\beta-\mathrm{CD}$ cavity, and moves freely in the aqueous medium. For the $S^{\prime} 1, S^{\prime} 2$ and $S^{\prime} 3$ simulations, this occurs 37,48 and $33 \%$ of the simulation period, respectively. Moreover, even when within the cavity the guest molecule is constantly in perpetual movement and adopts a fairly large number of conformations, indicating its instability inside. 


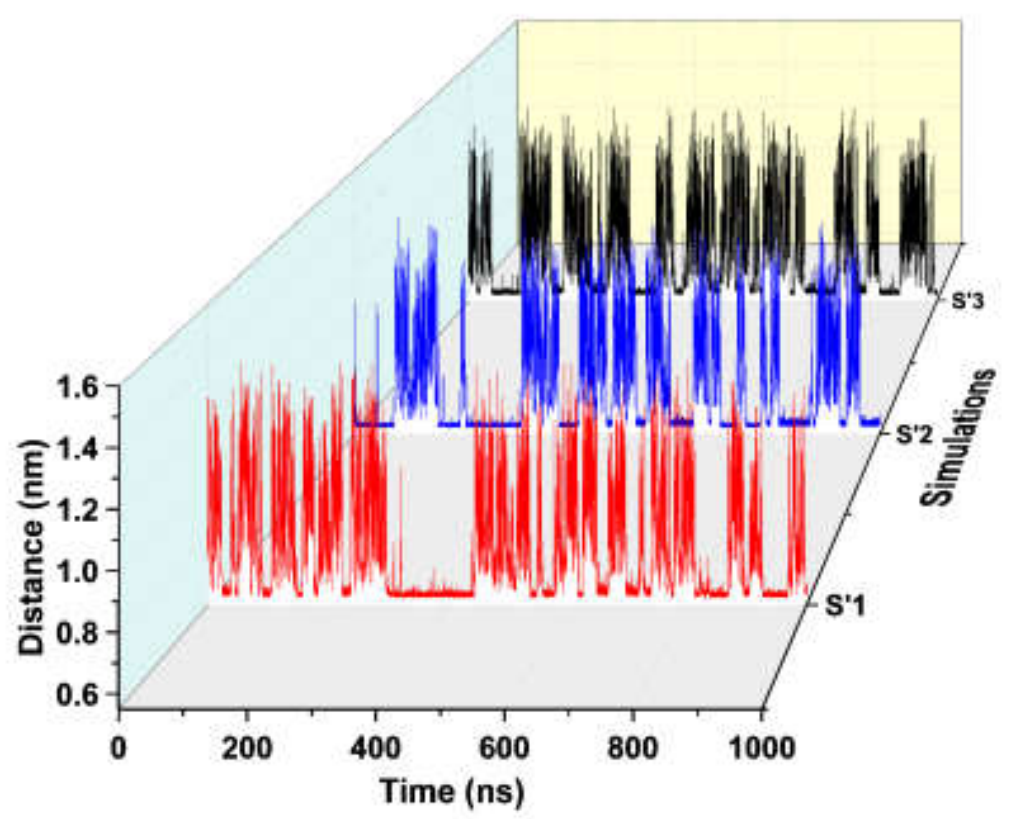

Figure 4. The variation of the distance between the mass centers of $\mathrm{ASA}^{-}$and $\beta-\mathrm{CD}$ during the three simulations.

From these simulations, three key points are highlighted: (i) the complexation process is reversible binding (i.e. the guest molecule can enter and exit the cavity several times) (ii) ASApenetrates inside the cavity through both the wider or the narrower rim; (iii) the inclusion complex is less favorable in aqueous medium because ASA- $^{-}$spends more time outside the cavity.

In order to quantify these observations we determined $k_{o n}, k_{o f f}, K$ and $\Delta G^{\circ}$ for ASA: $\beta-\mathrm{CD}$ and ASA: $\beta-C D$ complexes by using the Eqs. $(2,3,4,5$, and 6$)$ respectively. 
Table 1. Constant rate of association $k_{o n}$, constant rate of dissociation $k_{o f f}$, constant of association $K$ and association free energies, $\Delta G^{\circ}$, of ASA: $\beta-C D$ and ASA-: $\beta-C D$ complexes

\begin{tabular}{|c|c|c|c|c|c|}
\hline & & $\begin{array}{c}k_{o n} \\
\left(10^{9} / \mathrm{s} . \mathrm{M}\right)\end{array}$ & $\begin{array}{c}k_{o f f} \\
\left(10^{6 / s}\right)\end{array}$ & $\begin{array}{c}K \\
(1 / \mathrm{M})\end{array}$ & $\begin{array}{c}\Delta G^{\circ} \\
(\mathrm{kcal} / \mathrm{mol})\end{array}$ \\
\hline \multirow[t]{4}{*}{ ASA: $\beta-C D$} & Calculated & 6.25 & 1.99 & 3036 & -4.74 \\
\hline & & 7.21 & 1.31 & 549 & $-3.74^{\mathrm{a}}$ \\
\hline & Experimental & - & - & 149 & $-2.78^{b}$ \\
\hline & & - & - & 210 & $-3.15^{c}$ \\
\hline \multirow[t]{2}{*}{ ASA-: $\beta-C D$} & Calculated & 1.54 & 45.58 & 35.4 & -2.09 \\
\hline & Experimental & 0.78 & 15.2 & 51 & $-2.33^{a}$ \\
\hline
\end{tabular}

* a from reference $38,{ }^{\mathrm{b}}$ from reference 53 and ${ }^{\mathrm{c}}$ from reference 23

The values of $k_{o n}$ and $k_{o f f}$ of ASA and ASA- displayed in Table 1 show that ASA is formed five times more than ASA-, whereas ASA- is dissociated about 23 times more than ASA, meaning that ASA form more favorable complex with $\beta$-CD than ASA-. This can be explained by the fact that the carboxylate anion in ASA- reduces the stability of the ASA-: $\beta-C D$ complex, and is easily attracted by water molecules. This is in agreement with a point of view of Fukahori et al. which stipulate that the smaller value of $k_{\text {off }}$ leads to the more stable inclusion complex, since $k_{\text {on }}$ value was not so sensitive to a structure of host and guest molecules [38].

The computed values of free energies of ASA: $\beta-C D$ and ASA-: $\beta-C D$ complexes reported in Table 1 confirms that ASA: $\beta-C D$ complex is more favorable than ASA-: $\beta-C D$ by an energy difference of $2.65 \mathrm{kcal} / \mathrm{mol}$.

Also, as the value of free energy of ASA-: $\beta-C D$ complex is negative and equal to -2.09 this indicate that the process of its entrance in cavity is favorable despite having a strongly hydrophilic group. Confirming then that the hydrophobicity of the phenyl ring in ASA- is so high, that once it is close to the entrance of the $\beta$-CD cavity it gets inside.

Globally, all the obtained values of $\Delta \mathrm{G}^{\circ}$ agreed reasonably with experimental data in view of the difference in the experimental results for a given parameter because of their dependence on the 
used method (spectroscopic, analytic, ultrasound) and the operational conditions ( $\mathrm{pH}$, medium) $[23,38,53]$.

\subsection{Biased molecular dynamic simulation}

The free energy profile along the host-guest centroïds -to- centroïds distance $(\xi)$ was estimated using umbrella sampling combined to WHAM analysis. The calculated potentials of mean force (PMF) of the two studied complexes are depicted respectively in Figures 5 and 7.

\subsubsection{Thermodynamics of inclusion of ASA: $\beta-C D$ complex}

Figure 5 shows clearly that the inclusion of ASA inside the $\beta-\mathrm{CD}$ cavity is energetically favorable for both orientations because the free energy decreases to reach minima near the center of cavity.

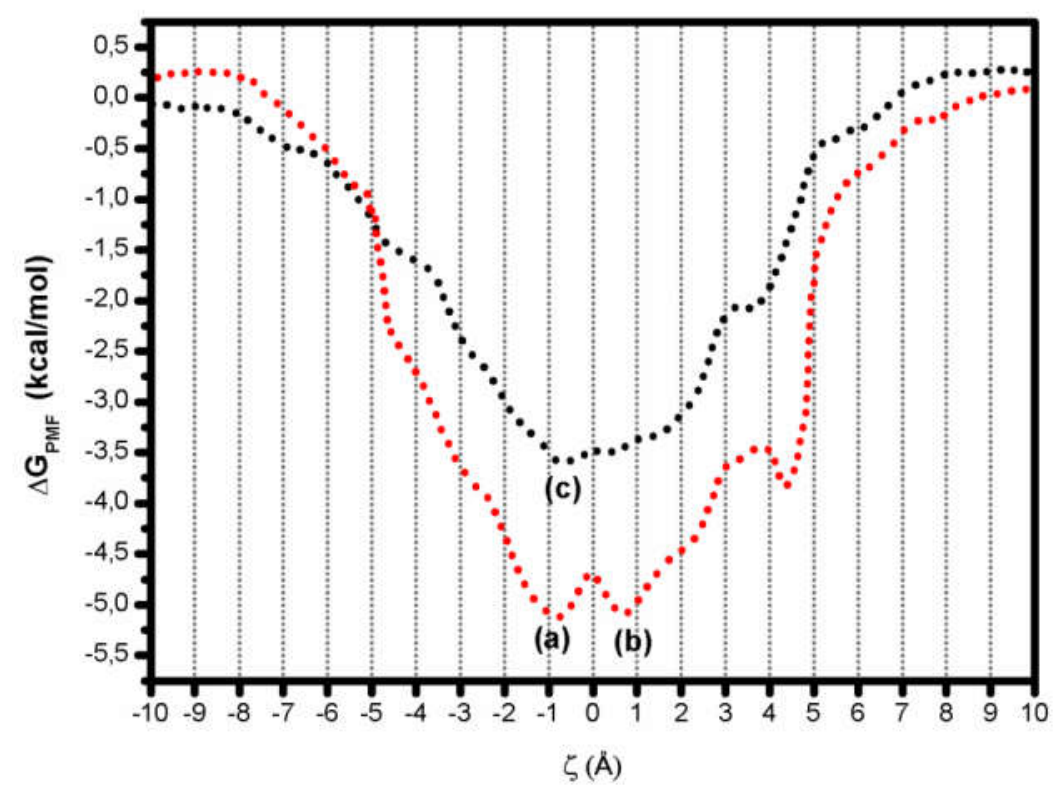

Figure 5. The free energy profiles from PMF calculations for the ASA: $\beta-C D$ complex, orientation A (red), orientation B (black)

The general aspect of the PMF profiles of orientation A show two local minima with little barrier separating them; whereas for orientation B a single less deep minimum in the PMF curve. Thus, the free energy of orientation A decreases as ASA approaches $\beta-C D$, leading to the first minimum around $-1 \AA(-0.6 \AA$ for orientation $B)$ with a free energy of $-5.1 \mathrm{kcal} / \mathrm{mol}(-3.5$ $\mathrm{kcal} / \mathrm{mol}$ for orientation $\mathrm{B})$. The structure of the first minimum for orientation $\mathrm{A}$ is shown in 
Figure 6a. It shows clearly that phenyl ring is deeply embedded inside the cavity on the wider rim, while the acetyl and carboxylic groups are located on the narrow rim, and only the methyl group is located outside the cavity. The structure of the minimum of orientation B shown in Figure $6 \mathrm{c}$ shows that the phenyl ring is inserted inside the cavity and all other parts of ASA are located outside the $\beta-\mathrm{CD}$ cavity. As ASA penetrates deeper in the $\beta-\mathrm{CD}$ cavity, a small energy barrier is located between (a) and (b) at $0 \AA$ with an energy height of $0.5 \mathrm{kcal} / \mathrm{mol}$. For orientation B no barrier is detected.

As ASA- penetrates deeper in the $\beta$-CD cavity, a second minimum for orientation $\mathrm{A}$ is located around $0.7 \AA$ with a free energy of $-5.13 \mathrm{kcal} / \mathrm{mol}$. The structure of the second minimum is shown in Figure $6 \mathrm{~b}$. The phenyl ring is buried in the $\beta-\mathrm{CD}$ cavity while all other parts of ASA are located on wider rim. It is interesting to note that the conformation of ASA inside the cavity is altered compared to its conformation in the first minimum position so that the two functional groups will be positioned on the opposite side. In terms of energy, when ASA leaves the $\beta-\mathrm{CD}$ cavity the energy increases progressively until it reaches a plateau when there is no interaction between ASA and $\beta-C D$.

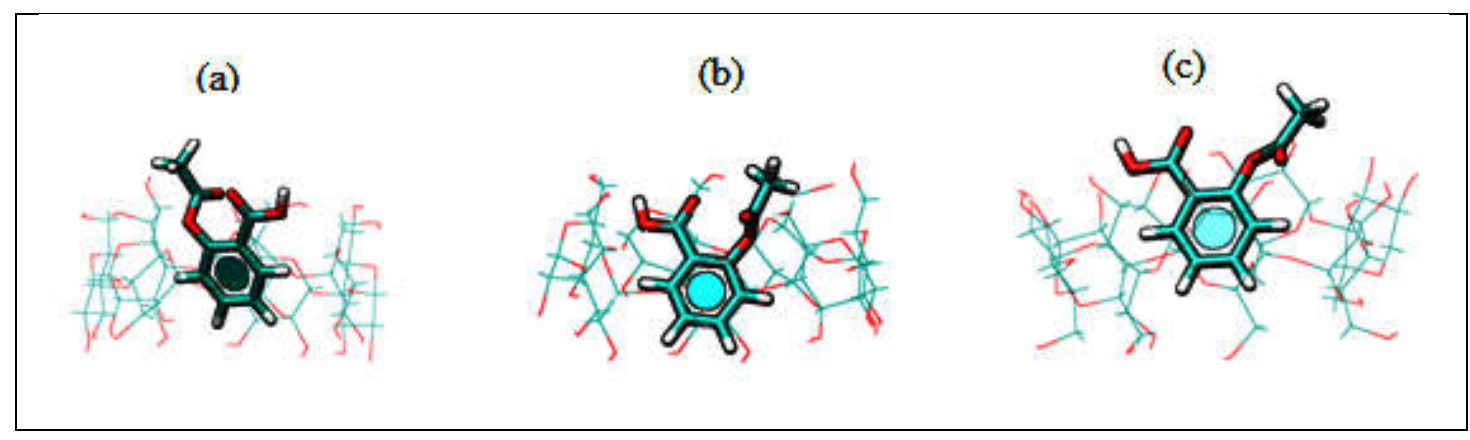

Figure 6. Snapshot of ASA: $\beta-C D$ complex of orientation A; (a) $1^{\text {st }}$ minimum (b) $2^{\text {nd }}$ minimum (c) minimum of orientation $B$

The energy difference between the two orientations was found to be equal to $2.0 \mathrm{kcal} / \mathrm{mol}$, suggesting that orientation $\mathrm{A}$ is more thermodynamically favored compared to B. From a structural point of view, the structural difference between them is that in orientation $A$ the inclusion is complete whereas in orientation B it is partial. Also, the ester and carboxylic groups are closer to the rim of $\beta-\mathrm{CD}$ in orientation $\mathrm{A}$ than $\mathrm{B}$. This orientation leads to stabilizing 
interactions with $\beta \mathrm{CD}$. One of the things we also notice that the phenyl ring on the wider rim of the cyclodextrin is ideally positioned to prevent close contacts with $\beta-\mathrm{CD}$.

\subsubsection{Thermodynamics of inclusion of $A S A^{-}$with $\beta-C D$}

The PMF profiles for the transfer of $\mathrm{ASA}^{-}$through $\beta-\mathrm{CD}$ according to the orientation $\mathrm{A}$ and $\mathrm{B}$ are plotted in Figure 7. It appears that both orientations provide almost the same shape of PMF profile as well as the same position of the maxima. The only significant difference observed is the depth of $\Delta \mathrm{GPMF}_{\mathrm{P}}$, which is about $1 \mathrm{kcal} / \mathrm{mol}$ deeper in orientation A compared to B. Figure 7 shows clearly that the inclusion is favorable up to points of 2 or $-2 \AA$ which become unfavorable.

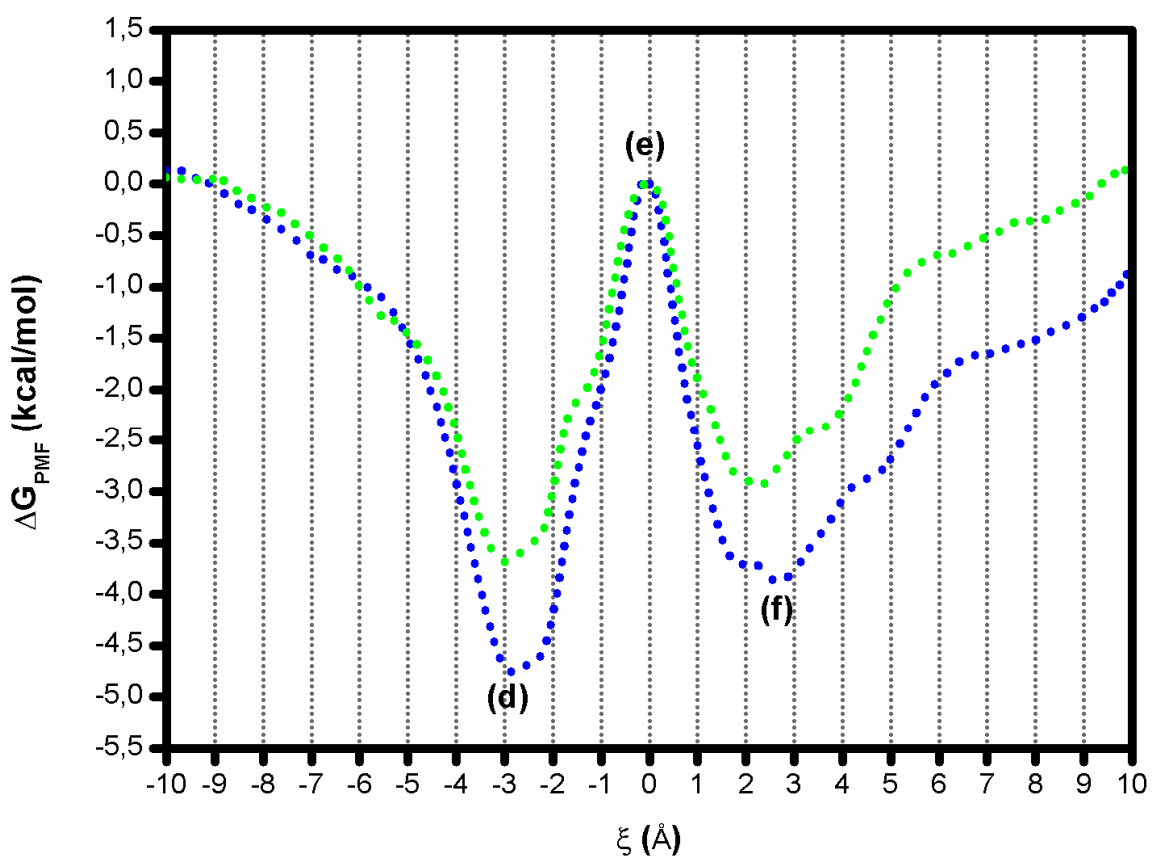

Figure 7. The free energy profiles from PMF calculations for the ASA $: \beta-C D$ complex, orientation A (blue), orientation B (green)

The free energy decreases as ASA- approaches $\beta-\mathrm{CD}$, leading to the first minimum at ca. - $2.9 \AA$ ( $3 \AA$ for orientation B) with a free energy stabilization of $-4.7 \mathrm{kcal} / \mathrm{mol}$ for orientation A (-3.7 $\mathrm{kcal} / \mathrm{mol}$ for $\mathrm{B}$ orientation). The structure of this stationary point of orientation $\mathrm{A}$ is presented in Figure $8 \mathrm{~d}$. This revealed that the inclusion is partial and concerns only the phenyl ring which is located inside the cavity and the rest of the molecule is located outside. As ASA-penetrates deeper in the $\beta$-CD cavity, an energy barrier appears of ca. $-4.8 \mathrm{kcal} / \mathrm{mol}$ for orientation A (3.9 $\mathrm{kcal} / \mathrm{mol}$ for orientation B). Now a maximum is located at $0 \AA$ for both orientations revealing a 
thermodynamically unfavorable state in the region of the center of $\beta$-CD. The structure of this stationary point is shown in Figure 8e. ASA- is deeply embedded inside the cavity leaving only the methyl group outside. In fact, the carboxylate anion inside the cavity induces unfavorable electrostatic interactions between negative charge of $\mathrm{ASA}^{-}$and the electric field created by the glycosidic oxygen atoms of $\beta$-CD.

The second minima occurs around $+2.6 \AA$ for orientation A ( $2 \AA$ for orientation B) with a free energy lowering of ca. $-3.7 \mathrm{kcal} / \mathrm{mol}$ for orientation A $(-2.9 \mathrm{kcal} / \mathrm{mol}$ for orientation $\mathrm{B})$. The structure of this stationary point is shown in Figure $8 \mathrm{f}$. From a structural point of view we notice the rotation of the guest molecule compared to its orientation at the beginning of the unbiased simulation. This rotation allows the phenyl ring to be inside the cavity instead of the outside, thereby promoting stabilizing hydrophobic interactions within the cavity. Subsequently, when

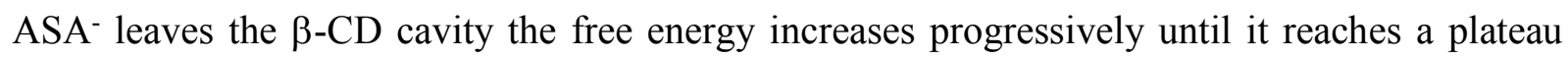
when the two chemical species no longer interact.

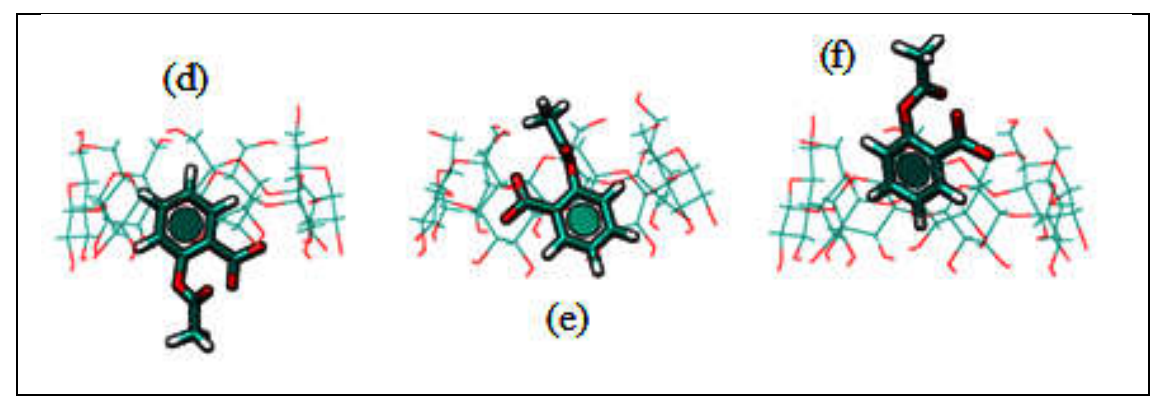

Figure 8. Specific conformations of ASA: $\beta-\mathrm{CD}$ complex of the orientation A

In this section, the study shows that the presence of the maximum in PMF calculations in the central region precludes an embedded inclusion. While the presence of two minima at ca. -2.6 and $2.9 \AA$ is synonym of a favorable inclusion process. As the inclusion is allowed only in the region energetically favorable, so it can be only partial and incomplete. These results are in agreement with those observed in our unbiased simulation highlighted in the first section and as well as with experimental results obtained by several authors who found that the inclusion is embedded with ASA and partial with ASA- [54-55]. 


\subsubsection{Association constants and association free energies.}

The association constant may be obtained by integrating the PMF along the ordering parameter, $\xi$, to the limit of association of ASA/ASA- and $\beta-\mathrm{CD}[56]$.

$$
K=\pi N_{A} \int r_{a v e}^{2} e^{\frac{-\Delta G(\xi)}{R T}} d \xi \cdots \cdots \cdots(6)
$$

Where $N_{A}$ is Avogadro's number and $\mathrm{R}$ is ideal gas constant. $r_{\text {ave }}$ is the average radius of the cross section in each bin, which evidently varies with $\xi$. The integration limit is the interval over which host-guest association is witnessed. The space integration over $\xi<0$ corresponds to the reference atom of ASA/ASA- by the wide entrance of $\beta-\mathrm{CD}$, whereas for $\xi>0$, this atom is by the narrow entrance of $\mathrm{CD}$. From the expression of $K$, the association free energy may be recovered through equation (5).

Table 2. The computed association constants and association free energies of ASA: $\beta-C D$ and ASA-: $\beta-C D$ complexes in A and B orientation

\begin{tabular}{ccccc}
\hline & Orientation & $\mathrm{K}\left(\mathrm{M}^{-1}\right)$ & $\Delta \mathrm{G}^{\circ}(\mathrm{kcal} / \mathrm{mol})$ & Difference $\left(\Delta \mathrm{G}^{\circ}\right)$ \\
\hline ASA: $\beta-\mathrm{CD}$ & A & 56.88 & -2.39 & 1.9 \\
& B & 5.10 & -0.49 & \\
ASA-: $\beta-\mathrm{CD}$ & A & 11.95 & -1.47 & 0.8 \\
& B & 3.10 & -0.67 & \\
\hline
\end{tabular}

The computed association constants and association free energies of ASA: $\beta-C D$ and ASA-: $\beta-C D$ complexes in A and B orientation are reported in Table 2.

Table 1 shows that the association free energies of ASA: $\beta$-CD complex are respectively equal to -2.39 and $-0.49 \mathrm{kcal} / \mathrm{mol}$ for $\mathrm{A}$ and $\mathrm{B}$ orientation; and are equal to -1.47 and $-0.67 \mathrm{kcal} / \mathrm{mol}$ in $\mathrm{A}$ and $\mathrm{B}$ orientations for ASA-: $\beta-\mathrm{CD}$ complex. This means that orientation $\mathrm{A}$ is more favorable than B for both complexes with $1.9 \mathrm{kcal} / \mathrm{mol}$ (neutral complex) and $0.8 \mathrm{kcal} / \mathrm{mol}$ (ionic complex) energy differences. These results are in the same direction as those obtained in the unbiased simulations whose orientation $\mathrm{A}$ is found to be more prominent than $\mathrm{B}$.

At last, we noted that the association free energies of both complexes obtained from PMF calculations are higher to those obtained from unbiased simulation but remains globally in the same order as those obtained experimentally. All the obtained values of $\Delta \mathrm{G}^{\circ}$ agreed reasonably 
with experimental data in view of the difference in the experimental results because of their dependence on the used method (spectroscopic, calorimetric, and ultrasonic) and the operational conditions ( $\mathrm{pH}$, medium). Thus, the results of unbiased simulation based on kinetic approach are closer to those obtained with ultrasonic method which also based on the same approach; while the results of biased simulation based on thermodynamic approach are closer to those obtained with calorimetric method which also based on thermodynamic approach.

\subsection{Hydrogen bonding}

Furthermore, we focused our main attention on the formation of the dynamic H-bonds during the formation process of the inclusion complex in order to provide dynamic picture of the interaction between the host and guest molecules. This can be achieved by using molecular dynamic simulation which can detect $\mathrm{H}$ bonds that occur during the complexation process. Since the simulations of the same complex gave similar results; and for the sake of simplicity we only presented the results of the H-bonds analysis of the simulations S3 and $\mathrm{S}^{\prime} 3$.

A hydrogen bond is considered as formed during the simulation when the donor-acceptor distance is less than $3.0 \AA$ and an angle cutoff equal to $20^{\circ}$. All H-bonds between $\beta$-CD and ASA/ASA ${ }^{-}$molecules are analyzed as well as those between $\beta-\mathrm{CD}$ and water molecules. Based on these criteria, we found 39 acceptors and 22 donor atoms for aspirin: $\beta-C D$ complex, and 39 acceptors and 21 donor atoms for ASA-: $\beta-C D$ complex.

\subsubsection{H-bonds of ASA: $\beta-C D$ complex}

The number of $\mathrm{H}$ bonds in the ASA: $\beta-\mathrm{CD}$ complex during the last $800 \mathrm{~ns}$ of the simulation that occur between ASA and $\beta-\mathrm{CD}$ as well as between ASA and water are displayed in Fig. 9.
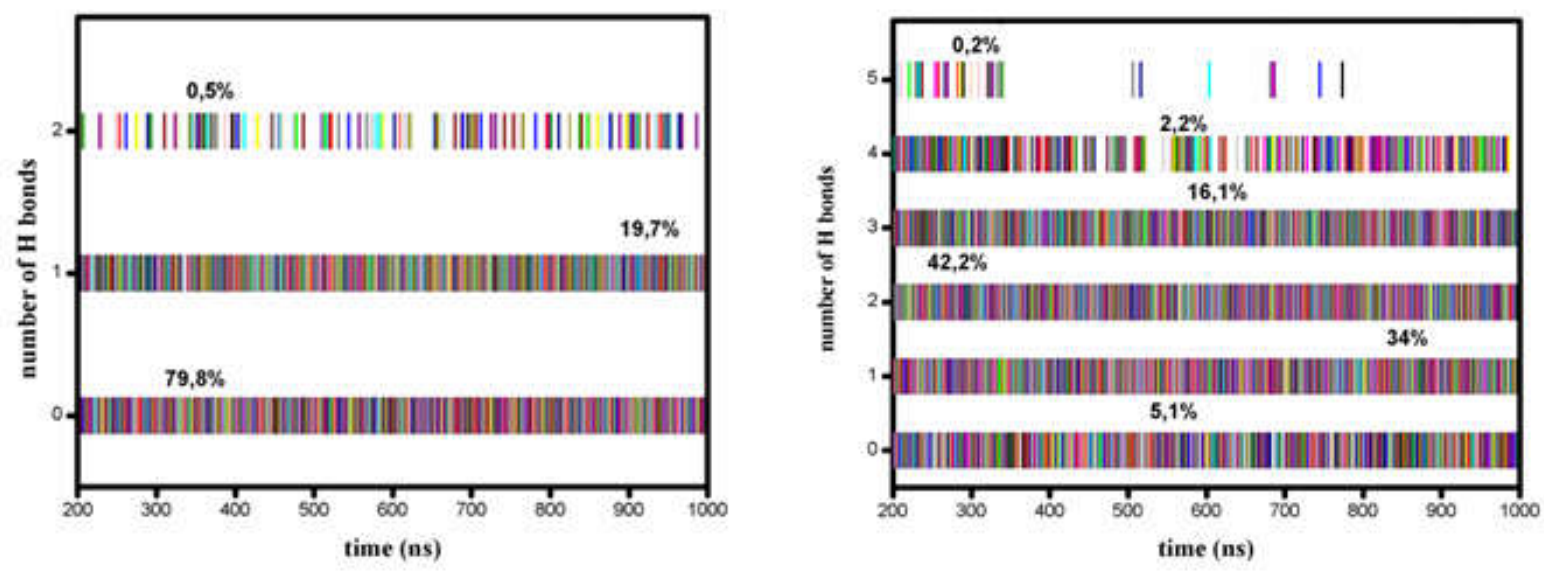
Figure 9. The number of H-bonds between ASA and $\beta-\mathrm{CD}$ (left) and between ASA and water (right)

As can be seen from Figure 9 (left), two H-bonds were detected during the simulation period, period in which ASA is totally inside the $\beta$-CD cavity. One hydrogen bond is observed during $19.7 \%$ of the simulation period in an intermittent manner and the second one is rarely detected having insignificant percentage of ca. $0.5 \%$ of the simulation period. This means that during about $79.8 \%$ of the simulation period the inclusion complex is formed without the establishment of H-bonds between ASA and $\beta$-CD. As a result, firstly, the inclusion complex doesn't necessarily need the H-bond interaction between ASA and $\beta-C D$; secondly, they are frequently formed and broken throughout the simulation probably due of the freedom of aspirin movement inside the cavity.

ASA can also establish H-bonds with water molecules. In Figure 9 (right) are depicted the number of H-bonds between ASA and water molecules during the last $800 \mathrm{~ns}$ of the simulation period. Thus, we observed most of the time at least one hydrogen bond between ASA and water molecules. Otherwise, during only $5.1 \%$ of the simulation period, water and ASA molecules are not linked by $\mathrm{H}$ bonds. Hence, these interactions between water and ASA are important for the stability of the inclusion complex in addition to its hydrophobic activity. Explicitly, one H-bond is detected during $34 \%$ of the simulation period, two H-bonds during $42.2 \%$, three $\mathrm{H}$-bonds during $16.1 \%$ and rarely with four and five $\mathrm{H}$ bonds.

In order to investigate more specifically the formation of H-bonds between ASA and $\beta-C D$ and also between ASA and water molecules, intermolecular radial distribution (RDFs) for the relevant atom-atom interactions, and the corresponding coordination number were calculated.

\subsubsection{H-bonds between ASA and $\beta$-CD}

The donor character of ASA given by the carboxylic hydrogen atom H5 is highlighted on the orientation A by the establishment of one H-bond with $\mathrm{O} 20$ oxygen atom of one of the primary hydroxyl group. The RDF curve for $\mathrm{H} 5 \cdots \mathrm{O} 20$ displayed in Figure 10 (left) show an intense peak located at ca. $2.45 \AA$ which extends from 2 to $3 \AA$. The coordinate number for $\mathrm{H} 5 \cdots \mathrm{O} 20$ derived from the $\mathrm{H} 5 \cdots \mathrm{O} 20 \mathrm{RDF}$ is estimated by integrating the first peak up to the second minimum. It is found equal to ca. 0.7 which is an indicative of a moderate $\mathrm{H}$-bond. 

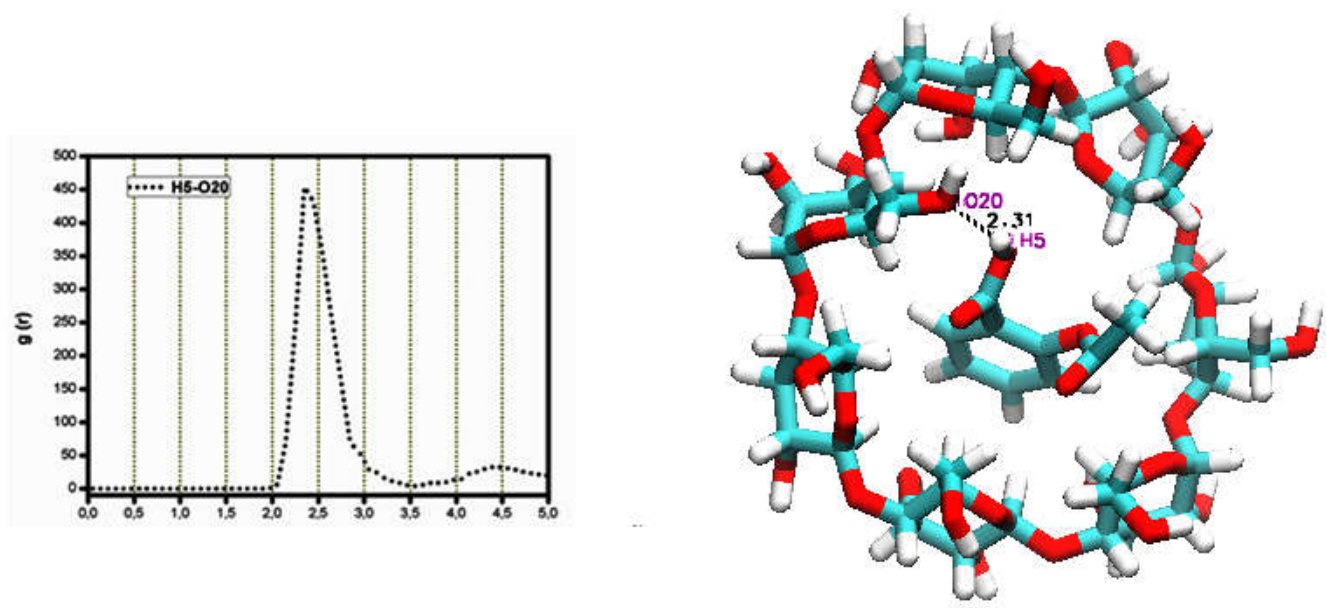

Figure 10. The RDF of $\mathrm{H} 5 \cdots \mathrm{O} 20 \mathrm{H}$-bond (left) and the snapshot extracted at $781 \mathrm{~ns}$ (right) of S3 simulation

The snapshot extracted at $239 \mathrm{~ns}$ displayed in Figure 10 (right) correspond to a geometry representing this state. It shows that $\mathrm{H} 5 \cdots \mathrm{O} 20 \mathrm{H}$-bond is located in orientation A with a distance of $2.75 \AA$ between the two involved atoms. This interaction likely favored by the orientation of the $\mathrm{O} 20-\mathrm{H} 60$ hydroxyl group of the $\beta-\mathrm{CD}$ which is the only one that pointed towards the interior of $\beta-\mathrm{CD}$, in direction of the guest molecule. As the Gibbs free energy difference between the two orientations estimated previously equal to ca. $2.0 \mathrm{kcal} / \mathrm{mol}$, a typical value of a moderate $\mathrm{H}$-bond for which energies vary between 1 and $2 \mathrm{kcal} / \mathrm{mol}$. This can be attributed to this H-bond interaction which is probably the cause of shift of the balance in favor of the orientation A.

\subsubsection{H-bond between ASA and water molecules}

We have also computed radial distribution functions between the hydrogen carboxylic atom and the four oxygen atoms of ASA and water molecules. These RDFs are shown in figure 11. 


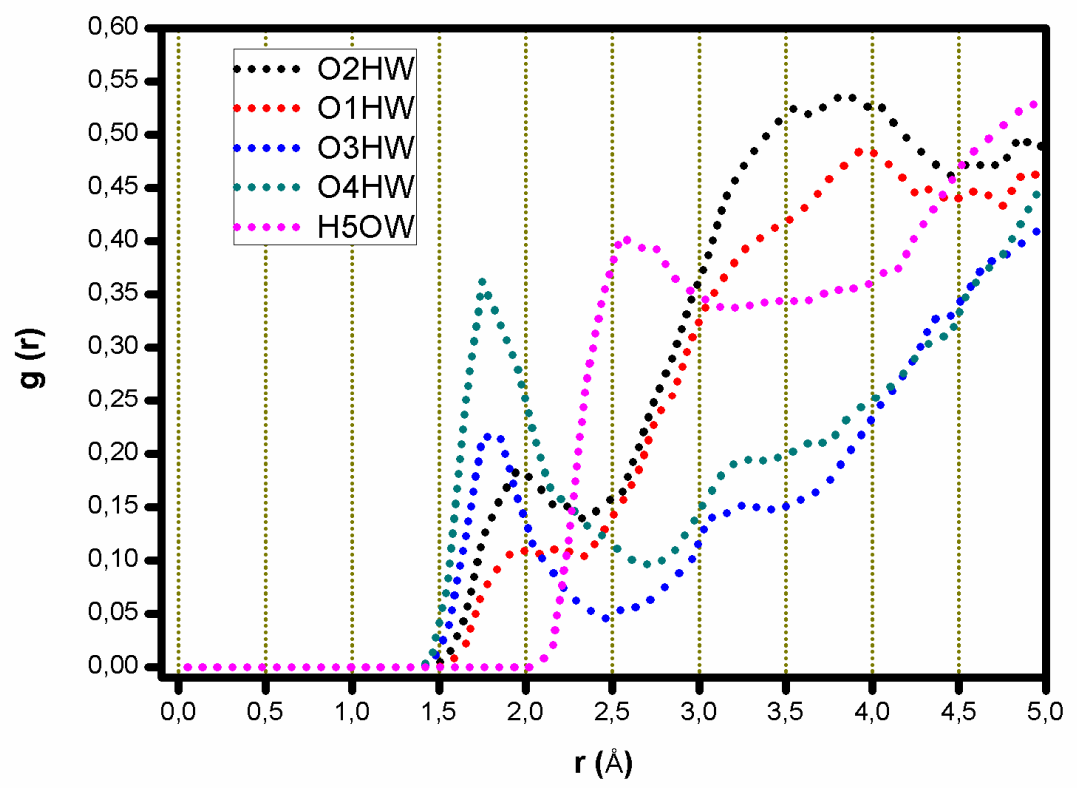

Figure 11. The RDFs between ASA and water

Thus, the RDF of H5 $\cdots$ OW shows a band with a maximum at $2.55 \AA$ without a clear minimum. The coordination number evaluated is equal ca. 1, synonym to a stable H-bond.

The RDFs curves O3 $\cdots \mathrm{HW}$ and $\mathrm{O} 4 \cdots \mathrm{HW}$ are shown with blue and green respectively, and those of $\mathrm{O} 1 \cdots \mathrm{HW}$ and $\mathrm{O} 2 \cdots \mathrm{HW}$ with red and black respectively (see Figure 1 for the numbering of oxygen atoms).

We notice a remarkable difference between the RDFs for O3 $\cdots \mathrm{HW}$ and $\mathrm{O} 4 \cdots \mathrm{HW}(\mathrm{O} 3$ and $\mathrm{O} 4$ keto oxygen atoms) on one hand and for $\mathrm{O} 1 \cdots \mathrm{HW}$ and $\mathrm{O} 2 \cdots \mathrm{HW}$ (O1 and $\mathrm{O} 2$ oxygen atom), on the other hand. Thus, the RDFs for $\mathrm{O} 3 \cdots \mathrm{HW}$ and $\mathrm{O} 4 \cdots \mathrm{HW}$ show a first peak centered at approximately 1.85 and $1.75 \AA$ respectively. The coordinate number derived from the $\mathrm{O} 3 \cdots \mathrm{HW}$

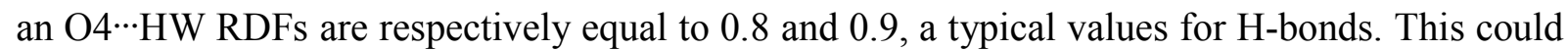
be considered as evidence of the formation of stable $\mathrm{H}$-bond between water hydrogen atoms and the $\mathrm{O} 3$ and $\mathrm{O} 4$ keto oxygen atoms of ASA.

However, both of $\mathrm{O} 1 \cdots \mathrm{HW}$ and $\mathrm{O} 2 \cdots \mathrm{HW}$ RDFs do not show a true peak at short intermolecular distances but rather a shoulder of low amplitude at ca. 2.0 $\AA$. The coordination numbers of both $\mathrm{O} 1$ and $\mathrm{O} 2$ oxygen atoms were estimated approximately equal to 0.5 and 07 respectively. Also, RDFs shows a broader and weaker second peak for $\mathrm{O} 1 \cdots \mathrm{HW}$ an $\mathrm{O} 2 \cdots \mathrm{HW}$ located at ca. $4.0 \AA$ with a coordination number equal to 4 and 5 which corresponds to the oxygen atoms of water of 
the second aqueous salvation layer. This suggests that the $\mathrm{O} 1$ and $\mathrm{O} 2$ oxygen atoms can form weak and much longer transient hydrogen bond with water molecules.

So, it seems that the keto oxygen atoms have a high tendency to form stable and strong hydrogen bond with water molecules of the first salvation layer, while the $\mathrm{O} 1$ and $\mathrm{O} 2$ oxygen atoms interacts rather with water molecules of the second salvation layer.

\subsection{2 $A S A^{-}: \beta-C D$ complex}

The numbers of $\mathrm{H}$-bonds between $\beta-\mathrm{CD}$ and $\mathrm{ASA}^{-}$and between $\mathrm{ASA}^{-}$and water molecules during the last $800 \mathrm{~ns}$ of the simulation are displayed in figure 12. As expected, no H-bonds were observed between $\mathrm{ASA}^{-}$and $\beta-\mathrm{CD}$ during $98.5 \%$ of the simulation period; and only one intermittent $\mathrm{H}$ bond during $1.5 \%$ of the simulation period is detected. This can be interpreted obviously by the fact that $\mathrm{ASA}^{-}$spent much more time in aqueous medium than inside the $\beta-$ CD cavity.
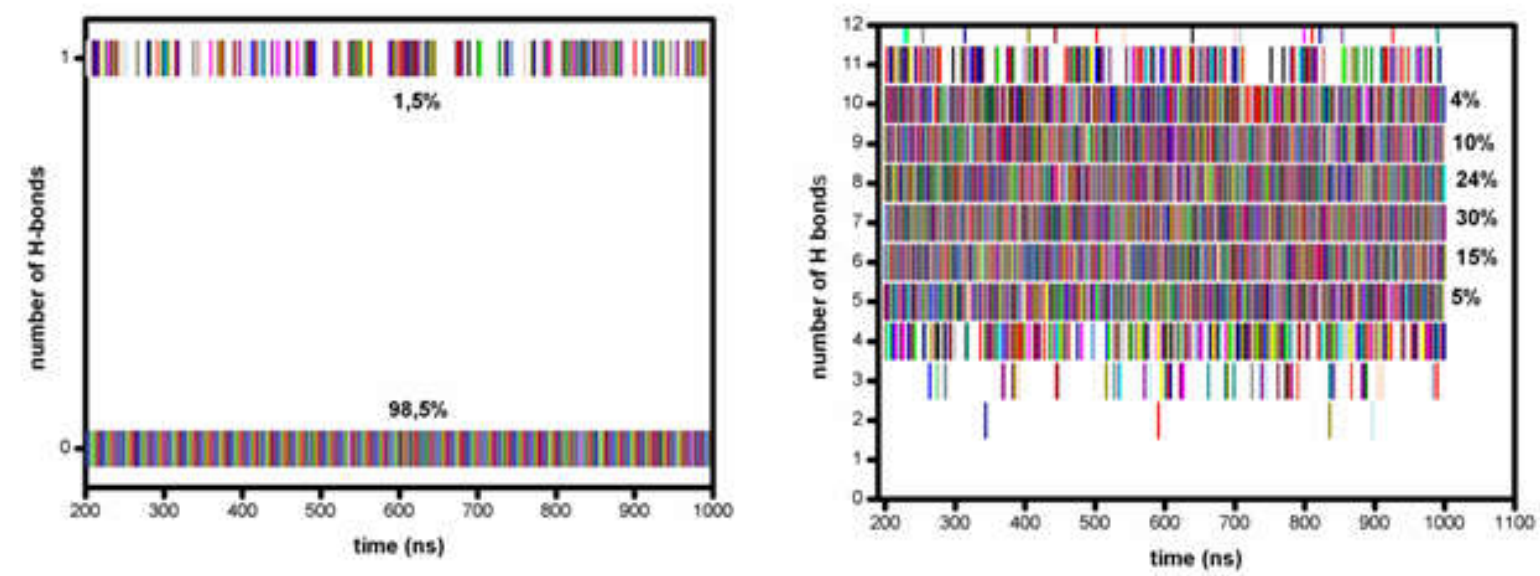

Figure 12. The number of $\mathrm{H}$-bonds between $\mathrm{ASA}^{-}$and $\beta-\mathrm{CD}$ (left) and between $\mathrm{ASA}^{-}$and water (right)

In Figure 12 (right) is displayed the number of $\mathrm{H}$-bonds between $\mathrm{ASA}^{-}$and water during the simulation period. It is shown that the number of $\mathrm{H}$-bonds varies widely during the simulation ranging from 2 to 12; some periods have moderate percentages. Thus, $7 \mathrm{H}$-bonds were observed during $30 \%$, 6 H-bonds during $24 \%$ and 8 H-bonds were detected during $15 \%$ of simulation period. For the others the percentages are equal or less than $10 \%$ of simulation period. 
This clearly confirms the hydrophilic affinity of $\mathrm{ASA}^{-}$to water molecules and explains why the process of its inclusion inside the $\beta-\mathrm{CD}$ cavity is less favorable.

\section{Conclusions}

Molecular dynamics applied to study the complexation process of ASA and ASA- with $\beta-\mathrm{CD}$ gives neutral complex more favorable than deprotonated one. In the neutral complex, the penetration occurs through the two entrances, and the more favorable orientation is that wherein the phenyl group is on the wider rim (orientation A). Moreover, the complexation process can be considered as no reversible binding. However, in the deprotonated complex, the penetration occurs through both of the wide and the narrow rims, and the complexation process is to be considered as reversible binding. The local minima structures obtained by PMF calculations show a deep inclusion in orientation A of ASA: $\beta-C D$ complex and partial in orientation $B$. However, for $\mathrm{ASA}^{-}: \beta-\mathrm{CD}$ complex the location of the carboxylate group in the central region of $\beta-C D$ causes a significant adverse interactions highlighted by an energy maximum in the PMF curve in this region indicating that the complete inclusion is unfavorable. Also, the two minima on the PMF curve state that the penetration is favorable through the two entrances as well as that the inclusion is partial.

The formation of H-bonds between $\beta-\mathrm{CD}$ and ASA was detected during $19.7 \%$ of the simulation period. RDF calculation shows that only the hydrogen carboxylic atom of ASA establishes a significant H-bond with $\beta$-CD contrary to the oxygen atoms of carboxylic and acetyl groups who do not establish any significant $\mathrm{H}$-bonds with $\beta$-CD. Although ASA is included inside $\beta-\mathrm{CD}$ cavity it keeps in contact with water molecules throughout $98.5 \%$ of simulation by establishing several $\mathrm{H}$ bonds.

Finally, it can be argued based on this dynamic picture of the complexation given in this study that the main driving forces in the inclusion process are hydrophobic/hydrophilic interactions, and are assisted by stabilizing H-bonds. 


\section{ACKNOWLEDGEMENTS}

The investigation was supported by the Algerian Ministry of higher education and scientific research and the research center in industrial technologies, Algiers (Algeria). Aix-Marseille University is gratefully acknowledged for an invited professor position (DK) + MESOCENTRE.

\section{AUTHOR INFORMATION}

Corresponding Author

*(K.D.E) E-mail (khatmi.djameleddine@gmail.com). Tel: (33) 783614430

Present address

Department of chemistry, University of Guelma, 24000, Algeria

Co-authors

Belgacem Bezzina (bezzinabelgacem@yahoo.fr)

Rayenne Djémil (messdjem@gmail.com)

Yannick Carissan (yannick.carissan@univ-amu.fr)

Pr Stéphane Humbel (stephane.humbel@univ-amu.fr)

\section{Author Contributions}

The manuscript was written through contributions of all authors. All authors have given approval to the final version of the manuscript. $\ddagger$ These authors contributed equally.

Notes

The authors declare no competing financial interest.

Funding Sources

The investigation was supported by the Algerian Ministry of higher education and scientific research and the research center in industrial technologies, Algiers (Algeria). DJ acknowledges Aix-Marseille University (France) for an invited professor position. 


\section{REFERENCES}

1. Szente L, Szejtli J (2004) Cyclodextrins as food ingredients, Trends in Food Science \& Technology 15(3):137-142.

2. Szente L, Szemán J, (2013) Cyclodextrins in analytical chemistry, Host-guest type molecular recognition. Analytical chemistry 85(17):8024-8030.

3. Lehn JM, (1988) Supramolecular chemistry - scope and perspectives: molecules supermolecules - molecular devices. J Inclusion Phenom 6 (4):351-396.

4. Marques HMC, (2010) A review on cyclodextrin encapsulation of essential oils and volatiles. Flav Frag J 25 (5):313-326.

5. Landy D, Tetart F, Truant E, Blach P, Fourmentin S, Surpateanu G, (2007) Development of a competitive continuous variation plot for the determination of inclusion compounds stoichiometry. J Incl Phenom Macro 57 (1-4):409-413.

6. Komiyama M, Bender ML, (1978) Thermodynamic studies of the cyclodextrin-accelerated cleavage of phenyl esters. J Am Chem Soc 100 (14):4576-4579.

7. Szejtli J, Bolla PE, Kajtar M (1982) The ß-cyclodextrin inclusion complex of menadione (vitamin K3). Pharmazie 37 (10):725-728.

8. Varady J, Wu X, Wang S, (2002) Competitive and reversible binding of a guest molecule to its host in aqueous solution through molecular dynamics simulation, Benzyl alcohol $/ \beta$ cyclodextrin system. The Journal of Physical Chemistry B 106 (18):4863-4872.

9. Challa R, Ahuja A, Ali J, Khar RK, (2005) Cyclodextrins in drug delivery, an updated review. AAPS PharmSciTech 6 (2):E329-E357.

10. Kanaka DDN, Prameela RA, Muneer AM, SaiKumar K, Kaushik JS, (2010) Cyclodextrins in pharmacy - an overview. Journal of Global Pharma Technology 2 (11):1-10.

11. Salustio PJ, Pontes P, Conduto C, Sanches I, Carvalho C, Arrais J, Marques, HMC, (2011) Advanced technologies for oral controlled release, cyclodextrins for oral controlled release. AAPS Pharm Sci Tech 12 (4):1276-1292. 
12. Yin JJ, Zhou ZW, Zhou SF, (2013) Cyclodextrin-based targeting strategies for tumor treatment. Drug Deliv Transl Res 3 (4):364-374.

13. Crini G, (2014) Review: a history of cyclodextrins. Chem Rev 114 (21):10940-10975.

14. Duchene D, Ponchel G, Bochot A, (2005) New uses of cyclodextrins. Eur J Pharm Sci 25: $\mathrm{S} 1-\mathrm{S} 2$.

15. Moore N, Ganse E V, Parc JML, Wall R, Schneid H, Farhan M, Verrière F, Pelen F, (1999) The PAIN Study: Paracetamol, Aspirin and Ibuprofen New Tolerability Study. Clin Drug Investig 18 (2): 89-98.

16. Impicciatore P, Pandolfini C, Casella N, Bonati M (1997) Reliability of Health Information for the Public on the World Wide Web: Systematic Survey of Advice on Managing Fever in Children at Home. BMJ 314 (7098):1875.

17. Chaudhary R, Bliden KP, Garg J, Mohammed N, Tantry U, Mathew D, Toth PP, Franzese C, Gesheff M, Pandya S, et al (2016) Statin Therapy and Inflammation in Patients with Diabetes Treated with High Dose Aspirin. J Diabetes Complications 30 (7):1365-1370.

18. Amory JK, Amory DW, (2007) Dosing Frequency of Aspirin and Prevention of Heart Attacks and Strokes. Am J Med 120 (4):e5.

19. Viola F, Lin-Schmidt X, Bhamidipati C, Haverstick DM, Walker WF, Ailawadi G, Lawrence MB, (2016) Sonorheometry Assessment of Platelet Function in Cardiopulmonary Bypass Patients: Correlation of Blood Clot Stiffness with Platelet Integrin $\alpha \operatorname{IIb} \beta 3$ Activity, Aspirin Usage, and Transfusion Risk. Thromb Res 138:96-102.

20. Rothwell PM, Wilson M, Elwin CE, Norrving B, Algra A, Warlow CP, Meade TW, (2010) Long-Term Effect of Aspirin on Colorectal Cancer Incidence and Mortality, 20-Year Follow-up of Five Randomised Trials. The Lancet 376 (9754):1741-1750.

21. Jiang MJ, Dai JJ, Gu DN, Huang Q, Tian L, (2016) Aspirin in Pancreatic Cancer, Chemopreventive Effects and Therapeutic Potentials. Biochim Biophys Acta 1866 (2):163176. 
22. Rainsford KD, (2004) Aspirin and Related Drugs, Ed CRC Press.

23. Castronuovo G, Niccoli M, (2013) Thermodynamics of Inclusion Complexes of Natural and Modified Cyclodextrins with Acetylsalicylic Acid and Ibuprofen in Aqueous Solution at $298 \mathrm{~K}$. Thermochim Acta 557:44-49.

24. Penner MJ, (1989) Aspirin Abolishes Tinnitus Caused by Spontaneous Otoacoustic Emissions, A Case Study, Arch Otolaryngol Head Neck Surg 115 (7):871-875.

25. Tee OS, Takasaki BK, (1985) The Cleavage of Aspirin by $\alpha$-and $\beta$-Cyclodextrins in Basic Aqueous Solution. Can J Chem 63 (12):3540-3544.

26. Carstensen J.T , Attarchi F , Hou X-P (1985). Decomposition of aspirin in the solid state in the presence of limited amounts of moisture. J. Pharm. Sci 74(7):741-745.

27. Tegge G, Szejtli J, (1982) Cyclodextrins and Their Inclusion Complexes (Cyclodextrine Und Ihre Einschlußkomplexe). Verlag Der Ungarischen Akademie Der Wissenschaften. Akadémiai Kiadó, Budapest 34 (11):395-395.

28. Dodziuk H, (2006) Front Matter, In Cyclodextrins and Their Complexes; Ed.; Wiley-VCH Verlag GmbH \& Co. KGaA pp I-XVII.

29. Cheng J, Wang Z, (2012) Synthesis of 5-Fluorouracil Acetic Acid Prodrugs of \#x0DF; Cyclodextrin at the Secondary Hydroxyl Side as Potential Colon-Specific Delivery Systems. In 2012 International Conference on Biomedical Engineering and Biotechnology :565-566.

30. Loftsson T Ólafsdóttir BJ, Friðriksdóttir H, Jónsdóttir S, (1993) Cyclodextrin Complexation of NSAIDSs: Physicochemical Characteristics. Eur J Pharm Sci 1 (2):95101.

31. Kalathil AA, Kumar A, Banik B, Ruiter TA, Pathak RK, Dhar S, (2015) New Formulation of Old Aspirin for Better Delivery. Chem Commun 52 (1):140-143.

32. Shende PK, Trotta F, Gaud RS, Deshmukh K, Cavalli R, Biasizzo M, (2012) Influence of Different Techniques on Formulation and Comparative Characterization of Inclusion 
Complexes of ASA with $\beta$-Cyclodextrin and Inclusion Complexes of ASA with PMDA Cross-Linked $\beta$-Cyclodextrin Nanosponges. J Incl Phenom Macrocycl Chem 74 (1-4): $447-454$.

33. Miranda JCD; Martins TEA, Veiga F, Ferraz HG, (2011) Cyclodextrins and Ternary Complexes: Technology to Improve Solubility of Poorly Soluble Drugs. Braz J Pharm Sci 47 (4):665-681.

34. Malenkovskaya MA, Grachev MK, Levina II, Nifant'ev EE, (2013) Amphiphilic Conjugates of $\beta$-Cyclodextrin with Acetylsalicylic and 2-(4-Isobutylphenyl)propionic Acids Russ J Org Chem 49 (12):1777-1782.

35. Nishioka F, Nakanishi I, Fujiwara T, Tomita K, (1984) The Crystal and Molecular Structure of the $\beta$-Cyclodextrin Inclusion Complex with Aspirin and Salicylic Acid. J Incl Phenom 2 (3-4):701-714.

36. Rajagopalan P, Penial P, (2013) Study of the spectral properties of inclusion complex of aspirin with hydroxy propyl $\beta$ - cyclodextrin. Int J Pharm Clin Sci Int 3 (4):24-28.

37. Nakai Y, Nakajima S-I, Yamamoto K, Terada K, Konno T,(1978) Effects of grinding on physical and chemical properties of crystalline medicinals with microcrystalline cellulose. III. Infrared spectra of medicinals in ground mixtures. Chemical and Pharmaceutical Bulletin 26(11): 3419-3425.

38. Fukahori T, Kondo M, Nishikawa S, (2006) Dynamic Study of Interaction between BetaCyclodextrin and Aspirin by the Ultrasonic Relaxation Method. J Phys Chem B 110 (9):4487-4491.

39. Sancho MI, Andujar S, Porasso RD, Enriz RD, (2016) Theoretical and Experimental Study of Inclusion Complexes of $\beta$-Cyclodextrins with Chalcone and 2',4'-Dihydroxychalcone. $\mathbf{J}$ Phys Chem B 120 (12):3000-3011.

40. Chen S, Han Z, Zhang D, Zhan J, (2014) Theoretical Study of the Inclusion Complexation of TCDD with Cucurbit[n]urils. RSC Advances 4 (94):52415-52422. 
41. Punkvang A, Saparpakorn P, Hannongbua S, Wolschann P, Beyer A, Pungpo P, (2010) Investigating the Structural Basis of Arylamides to Improve Potency against M. Tuberculosis Strain through Molecular Dynamics Simulations. Eur J Med Chem 45 (12):5585-5593.

42. Schönbeck, C. (2018). Charge Determines Guest Orientation: A Combined NMR and Molecular Dynamics Study of $\beta$-Cyclodextrins and Adamantane Derivatives. The Journal of Physical Chemistry B, 122(18), 4821-4827.

43. Tang, Z., \& Chang, C. E. A. (2017). Binding Thermodynamics and Kinetics Calculations Using Chemical Host and Guest: A Comprehensive Picture of Molecular Recognition. Journal of chemical theory and computation, 14(1), 303-318.

44. Lotz, S. D., \& Dickson, A. (2018). Unbiased molecular dynamics of 11 min timescale drug unbinding reveals transition state stabilizing interactions. Journal of the American Chemical Society, 140(2), 618-628.

45. Kerwin SM, (2010) ChemBioOffice Ultra 2010 Suite. J Am Chem Soc 132 (7):2466-2467.

46. Bolton EE, Wang Y, Thiessen PA, Bryant SH, (2008) Integrated Platform of Small Molecules and Biological Activities. In Annual Reports in Computational Chemistry; Spellmeyer, R. A. W. and D. C., Ed.; Elsevier (4):217-241.

47. Malde AK, Zuo L, Breeze M, Stroet M, Poger D, Nair PC, Oostenbrink C, Mark AE, (2011) An Automated Force Field Topology Builder (ATB) and Repository: Version 1.0. J. Chem Theory Comput 7 (12):4026-4037.

48. Schmid N, Eichenberger AP, Choutko A, Riniker S, Winger M, Mark AE, Gunsteren WFV (2011) Definition and Testing of the GROMOS Force-Field Versions 54A7 and 54B7. Eur Biophys J 40 (7):843-856.

49. Abraham MJ, Murtola T, Schul R, Páll S, Smith JC, Hess B, Lindahl E, (2015) GROMACS: High Performance Molecular Simulations through Multi-Level Parallelism from Laptops to Supercomputers. Software, 1-2:19-25. 
50. Van Der Spoel D, Lindahl E, Hess B, Groenhof G, Mark AE, Berendsen HJC, (2005) GROMACS: Fast, Flexible, and Free. J Comput Chem 26 (16):1701-1718.

51. Copeland, Robert A. Evaluation of enzyme inhibitors in drug discovery: a guide for medicinal chemists and pharmacologists. John Wiley \& Sons, 2013.

52. Roux, B. (1995). The calculation of the potential of mean force using computer simulations. Computer physics communications, 91(1-3), 275-282.

53. Dahab, Ali Aboel, and Dhia El-Hag. "Rapid analysis of NSAIDs binding to $\beta$-cyclodextrin using the simultaneous measurement of absorption and circular dichroism with a novel multi-cell low-volume device." Analytical and bioanalytical chemistry 404.6-7 (2012): 1839-1850.

54. Kitchin SJ, Halstead TK, (1999) Solid-state2H NMR Studies of Methyl Group Dynamics in Aspirin and Aspirin · $\beta$-Cyclodextrin. Appl Magn Reson 17 (2-3):283-300.

55. Choi HS, (1991) Structure Study of Inclusion Complex of ß Cyclodextrine and Aspirin. J. Pharm Investig 21 (4):223-230.

56. Zhang, H., Tan, T., Hetényi, C., and Van Der Spoel, D. (2013). Quantification of solvent contribution to the stability of noncovalent complexes. Journal of chemical theory and computation, $9(10), 4542-4551$. 